Claudia Garcia Serpa Osorio-de-Castro 1,2 Maurício Abreu Pinto Peixoto 3 Selma Rodrigues de Castilho 4

\section{Câmbio na assistência perinatal como determinante de consumo e diversidade de antiinfecciosos? Estudo em UTI neonatal no Rio de Janeiro}

\author{
Changes in perinatal care as a determinant \\ of the level and diversity of antiinfectives \\ use in a neonatal intensive care unit \\ in Rio de Janeiro, Brazil
}

1 Núcleo de Assistência Farmacêutica, Escola Nacional de Saúde Pública, Fundação Oswaldo Cruz. Rua Leopoldo Bulhões 1480 , sala 602 , Rio de Janeiro, $R J$ 21041-210, Brasil. claudia@iff.fiocruz.br 2 Serviço de Farmácia, Instituto Fernandes Figueira, Fundação Oswaldo Cruz. Av. Rui Barbosa 716, Rio de Janeiro, $R J$ 22250-020, Brasil. 3 Núcleo de Tecnologia Educacional para a Saúde, Universidade Federal do Rio de Janeiro. Cidade Universitária, Centro de Ciências da Saúde, Bloco A (térreo), Rio de Janeiro, $R J$ 21941-590, Brasil. mpeixoto@nutes.ufrj.br 4 Centro de Apoio à Terapia Racional pela Informação sobre Medicamentos, Faculdade de Farmácia, Universidade Federal Fluminense. Rua Mário Vianna 523, Niterói, RJ 24241-000, Brasil. selma@predialnet.com.br

\begin{abstract}
This study focuses on a five-year profile (1990-1994) in the utilization of antiinfectives in a neonatal intensive care unit (NICU) in Rio de Janeiro, Brazil. Total antiinfectives consumption, diversity, and possible determinants were analyzed. A descriptive, longitudinal, and retrospective drug utilization study design was adopted. Document research, observation, and semi-structured interviews provided information on the neonatal intensive care provided, and drug supply and daily hospital records provided data on antiinfectives use, based on the Anatomical Therapeutic Chemical (ATC) classification and the infant defined daily dose (DDDi) as units of measurement. The numerical results suggest an increase in total consumption of antiinfectives and a decrease in their diversity. Analyzed in view of the institutional profile and rational drug use, these results indicate that changes in the type of care provided during the study period were key determinants of antiinfectives use.
\end{abstract}

Key words Anti-Infective Agents; Perinatal Care; Drug Utilization

Resumo O perfil de utilização de antiinfecciosos, constando de consumo e diversidade dos fármacos empregados, bem como seus determinantes, foram estudados em um período de cinco anos, de janeiro de 1990 a dezembro de 1994, em duas enfermarias de uma unidade de terapia intensiva (UTI) neonatal do Rio de Janeiro. Para tanto, cumpriu-se estudo de utilização de medicamentos com desenho descritivo, longitudinal e retrospectivo. Como sistemática foram empregadas a classificação Anatomical Therapeutic Chemical (ATC) e a unidade de medida dose diária definida para lactentes (DDDi). Foram coletados dados de serviço, através de pesquisa documental, observações e entrevistas semi-estruturadas, e dados numéricos, através de consulta aos registros de fornecimento do Serviço de Farmácia e registros do censo hospitalar diário. Os resultados numéricos sugerem um incremento real do consumo total e a diminuição da diversidade no uso de antiinfecciosos no período. Analisados à luz da realidade institucional e do uso racional de medicamentos, indicam que mudanças na assistência perinatal ao longo do período foram determinantes para o perfil de utilização revelado.

Palavras-chave Antiinfecciosos; Assistência Perinatal; Uso de Medicametos 


\section{Introdução}

A expectativa universal em relação à terapêutica medicamentosa é de que ela deva ser conduzida de tal modo que os medicamentos prescritos realmente previnam, diagnostiquem, aliviem ou curem doenças. Essa é a proposta da utilização racional dos medicamentos: o medicamento cumprindo seu papel de instrumento terapêutico. O uso racional acontece quando primeiramente se estabelece a real necessidade de emprego do medicamento. Segue-se necessariamente uma prescrição adequada e responsável, atenta simultaneamente às evidências de eficácia e segurança do fármaco, à forma farmacêutica mais apropriada, e também à dose e ao período de duração do tratamento, disponibilidade no mercado e condições de acesso do paciente. O medicamento assim prescrito deve ter qualidade garantida, ser armazenado e dispensado em condições adequadas, sendo ainda indispensável orientação quanto à sua utilização. Por fim, a racionalidade inclui o pleno cumprimento do regime terapêutico (Osorio-de-Castro et al., 2000; WHO, 1987).

Muitos fatores influenciam os padrões de utilização de medicamentos. Hábitos culturais, práticas prescritivas, mercado farmacêutico, propaganda, vertentes de informação são fatores que circundam e influenciam aquele que seria considerado o mais importante determinante do uso: a necessidade real. Esses fatores caminham também longe das propriedades intrínsecas dos medicamentos, de sua eficácia e segurança e, possivelmente não raro, determinam padrões irracionais de utilização (Laporte et al., 1993). O exame dos padrões de uso de produtos medicamentosos visa ainda evidenciar aspectos pouco elucidados no próprio sistema ou nas unidades de saúde que o compõem.

De modo a promover o uso racional de medicamentos, a Organização Mundial de Saúde (OMS) lançou, há quase vinte e cinco anos, o conceito de medicamento essencial. São considerados essenciais aqueles que atendem às necessidades sanitárias da população, demonstrando eficácia e segurança, estando disponíveis em quantidade e qualidade adequadas, $\mathrm{e}$ ostentando custo compatível com as possibilidades de quem os adquire (Mosegui, 1999). Ao conceito de medicamento essencial, a OMS agregou a adoção de listas padronizadas de medicamentos e ainda de guias terapêuticos, como estratégias para implementação do uso racional. O objetivo não é o de restringir a oferta, mas de aumentar a efetividade da terapêutica, possibilitando ao prescritor, assim como aos demais profissionais de saúde, maior conhecimento sobre o fármaco com o qual está lidando no dia-a-dia, face à sua inserção clínica específica (Tognoni \& Lunde, 1993).

Uma das críticas ao sistema de medicamentos padronizados é o conservadorismo das listas; novidades terapêuticas não tendem a ser incorporadas. Essa suposta falha é, no entanto, uma das âncoras do sistema. O uso racional é também, de certo modo, uma função com componente temporal: quanto mais antigo um medicamento mais se sabe sobre seus efeitos, tanto em relação à segurança quanto à eficácia, pois terá sido utilizado por maior número de pessoas. Esse conservadorismo é portanto, até certo ponto, desejável (Fijn et al., 2000).

Em meados da década de 90, Sackett e colaboradores propõem o paradigma da Medicina Baseada em Evidência (MBE). Segundo os autores, o tratamento de cada paciente individual deve estar sempre embasado na melhor evidência clínica disponível naquele momento (Fuchs et al., 2000; Sackett et al., 2000). Sem anular a individualidade do paciente frente à visão de coletividade que pode emanar da literatura científica, a MBE fomenta a adoção do uso racional de medicamentos, quando aponta para a adoção de consensos e protocolos de utilização. Idealmente, na equipe de saúde cria-se um ambiente de conhecimento compartilhado, onde as ações terapêuticas de cada participante guardam semelhanças e objetivos comuns. Por isto, segundo Schneider $(1999,2001)$ e Kohn (2001) a determinação de padrões de assistência, a revisão e avaliação periódica de procedimentos constituem-se em estratégias recomendáveis.

Nos últimos trinta anos, houve crescente desenvolvimento da neonatologia como área de especialização e maior exigência de qualificação dos profissionais atuantes (Polack et al., 1998). A importância dada à segurança dos processos terapêuticos e ao incremento da qualidade da assistência, levou ao intenso desenvolvimento de protocolos e ao incentivo à adesão a condutas bem estabelecidas. Sua aplicabilidade tem se manifestado em diversos campos disciplinares, entre outros, no uso de antiinfecciosos, em condutas terapêuticas em pediatria e neonatologia e em cuidado intensivo (Albrant, 1999; Anand et al., 2001; Andersen \& Meberg, 1999; Fischer et al., 2000; Gindre et al., 2000; Jacobs, 2000; Natsch et al., 2000; Pons et al., 1999; Whyte et al., 2000).

O presente artigo, analisa a temática sob a perspectiva da utilização de medicamentos. Será que interferências na assistência perinatal - câmbio da clientela e evolução integrada da 
equipe de uma unidade de terapia intensiva (UTI) neonatal no Rio de Janeiro - podem ser determinantes no perfil de utilização de medicamentos? Como variáveis de estudo, escolheu-se a diversidade dos antiinfecciosos utilizados e a intensidade de seu consumo em um período de cinco anos, assim como a descrição de aspectos institucionais relevantes.

\section{Métodos}

O estudo cumpriu um desenho descritivo, retrospectivo, no período de 1990 a 1994, tendo como objeto as enfermarias Berçário de Alto Risco (BAR) e Berçário Intermediário (BI) do Departamento de Neonatologia do Instituto Fernandes Figueira (IFF).

\section{O Instituto Fernandes Figueira}

Unidade hospitalar terciária, congregando atividades de ensino, pesquisa e assistência, e privilegiando esta última, tem seu âmbito de ação na área materno-infantil.

\section{- Berçário de Alto Risco e o Berçário Intermediário}

O Departamento de Neonatologia desenvolve suas atividades em diversos setores, incluindo duas enfermarias de tratamento intensivo, o BAR e o BI. À época, estas enfermarias estavam abrigadas em duas salas com $70 \mathrm{~m}^{2}$ cada, totalizando cerca de vinte leitos, com um mínimo operante de 15 incubadoras aquecidas. O tempo médio de internação era de 21 a 22 dias.

No BI eram alojados os neonatos de risco intermediário que necessitavam de cuidados intensivos, fototerapia e acompanhamento de sinais vitais, mas que prescindiam de respirador, hidratação venosa ou nutrição parenteral. Tais crianças podiam ser amamentadas diretamente pela mãe. Já no BAR estavam recémnascidos de alto risco/baixo peso, com respiração assistida, hidratação venosa e/ou nutrição parenteral, acompanhamento de sinais vitais e parâmetros laboratoriais.

A equipe médica era composta por quatro médicos diaristas, três consultores, um plantonista e cerca de cinco residentes, com supervisão médica feita em rodízio mensal entre cinco dos profissionais mais antigos. A equipe de enfermagem contava com quatro enfermeiras plantonistas em rodízio. Cerca de seis auxiliares de enfermagem e uma auxiliar de conservação e asseio compunham diariamente a equipe.

\section{- Grupo terapêutico e seleção}

Elegeu-se o grupo terapêutico dos antiinfecciosos, com os seguintes critérios de seleção: (a) estar padronizado pelo IFF (fármaco e forma farmacêutica); (b) estar presente na listagem Anatomical Therapeutic Chemical (ATC) como antiinfeccioso (WHO, 1993); (c) possuir dose diária definida (DDD) listada, consoante com as formas farmacêuticas padronizadas para uso no hospital; (d) ter tido uso no BAR/BI no período de janeiro de 1990 a dezembro de 1994.

O sistema ATC de classificação de medicamentos e a unidade chamada DDD são ferramentas clássicas dos estudos de utilização de medicamentos, com a finalidade de homogeneizar resultados, tornando-os comparáveis (Osorio-de-Castro et al., 2000). Em estudos com população pediátrica menor de um ano, emprega-se o indicador chamado dose diária definida em lactentes (DDDi) correspondendo a 1/10 da DDD (Capellà, 1993; Matheson, 1991).

Dos 32 antiinfecciosos inicialmente listados, apenas 23 constituíram o grupo de estudo, segundo o critério (d) (Tabela 1$)$.

\section{Coleta de dados}

Dois tipos de dados, de diferente natureza, foram contemplados:

\section{a) Dados de serviço}

De modo a contextualizar o trabalho e avaliar os resultados, foram realizadas pesquisa documental e observação participante relativas às unidades de internação envolvidas, assim como entrevistas semi-estruturadas com profissionais da equipe do Departamento de Neonatologia, a saber, a responsável pelo Departamento, médicos e enfermeiros. As entrevistas foram individuais, na tentativa de obter validação cruzada das informações prestadas.

\section{b) Dados numéricos (relativos a medicamentos e à movimentação hospitalar)}

Por meio dos registros de fornecimento do Serviço de Farmácia (FIOCRUZ, 1994a), o consumo unitário mensal foi quantificado em número de DDDi de cada um dos antiinfecciosos para o período compreendido entre janeiro de 1990 e dezembro de 1994. Foram observadas as diferentes apresentações para a mesma forma farmacêutica. 
Tabela 1

Antiinfecciosos utilizados no Berçário de Alto Risco e Berçário Intermediário. Instituto Fernandes Figueira, janeiro de 1990 a dezembro de 1994.

\begin{tabular}{|c|c|c|c|c|}
\hline Antiinfeccioso & Via de administração & $\begin{array}{l}\text { Quantidade de princípio } \\
\text { ativo por forma farm. }\end{array}$ & Valor da DDD & Valor da DDDi \\
\hline Nistatina & O & $4 \mathrm{ME}$ & $1,5 \mathrm{ME}$ & 150TE \\
\hline Anfotericina B & $P$ & $50 \mathrm{mg}$ & $35 \mathrm{mg}$ & $3,5 \mathrm{mg}$ \\
\hline Cloranfenicol & $\mathrm{P}$ & $1.000 \mathrm{mg}$ & $3.000 \mathrm{mg}$ & $300 \mathrm{mg}$ \\
\hline Ampicilina & $\mathrm{O} / \mathrm{P}$ & $3.000 \mathrm{mg} / 500 \mathrm{mg}$ & $2.000 \mathrm{mg}$ & $200 \mathrm{mg}$ \\
\hline Carbenicilina & $\mathrm{P}$ & $1.000 \mathrm{mg}$ & $12.000 \mathrm{mg}$ & $1.200 \mathrm{mg}$ \\
\hline Benzilpenicilina & $P$ & $3.135 \mathrm{mg}$ & $3.600 \mathrm{mg}$ & $360 \mathrm{mg}$ \\
\hline Oxacilina & $P$ & $500 \mathrm{mg}$ & $2.000 \mathrm{mg}$ & $200 \mathrm{mg}$ \\
\hline Cefalexina & $\mathrm{O}$ & $1.500 \mathrm{mg}$ & $2.000 \mathrm{mg}$ & $200 \mathrm{mg}$ \\
\hline Cefalotina & $P$ & $1.000 \mathrm{mg}$ & $4.000 \mathrm{mg}$ & $400 \mathrm{mg}$ \\
\hline Cefoxitina & $P$ & $1.000 \mathrm{mg}$ & $6.000 \mathrm{mg}$ & $600 \mathrm{mg}$ \\
\hline Cefotaxima & $P$ & $500 \mathrm{mg}$ & $6.000 \mathrm{mg}$ & $600 \mathrm{mg}$ \\
\hline Ceftazidima & $\mathrm{P}$ & $1.000 \mathrm{mg}$ & $6.000 \mathrm{mg}$ & $600 \mathrm{mg}$ \\
\hline Ceftriaxona & $P$ & $500 \mathrm{mg}$ & $2.000 \mathrm{mg}$ & $200 \mathrm{mg}$ \\
\hline Imipenem & $P$ & $500 \mathrm{mg}$ & $2.000 \mathrm{mg}$ & $200 \mathrm{mg}$ \\
\hline Sulfametoxazol & $\mathrm{O}$ & $2.000 \mathrm{mg}$ & $2.000 \mathrm{mg}$ & $200 \mathrm{mg}$ \\
\hline Sulfadiazina & O & $500 \mathrm{mg}$ & $600 \mathrm{mg}$ & $60 \mathrm{mg}$ \\
\hline Eritromicina & $\mathrm{O}$ & $7.500 \mathrm{mg}$ & $1.000 \mathrm{mg}$ & $100 \mathrm{mg}$ \\
\hline Clindamicina & O & $600 \mathrm{mg}$ & $1.800 \mathrm{mg}$ & $180 \mathrm{mg}$ \\
\hline Gentamicina & $P$ & $10 \mathrm{mg} / 20 \mathrm{mg} / 80 \mathrm{mg}$ & $240 \mathrm{mg}$ & $24 \mathrm{mg}$ \\
\hline Amicacina & $P$ & $100 \mathrm{mg} / 250 \mathrm{mg}$ & $1.000 \mathrm{mg}$ & $100 \mathrm{mg}$ \\
\hline Vancomicina & $P$ & $500 \mathrm{mg}$ & $2.000 \mathrm{mg}$ & $200 \mathrm{mg}$ \\
\hline Metronidazol & $P$ & $500 \mathrm{mg}$ & $1.500 \mathrm{mg}$ & $150 \mathrm{mg}$ \\
\hline Isoniazida & $\mathrm{O}$ & $100 \mathrm{mg}$ & $300 \mathrm{mg}$ & $30 \mathrm{mg}$ \\
\hline
\end{tabular}

$\mathrm{ME}=$ milhão de unidades; $\mathrm{TE}=$ mil unidades; $\mathrm{mg}=$ miligramas; $\mathrm{O}=$ via oral; $\mathrm{P}=$ via parenteral;

DDD = dose diária definida; $\mathrm{DDDi}=$ dose diária definida em lactentes.

Foram coletados os dados mensais, no período do estudo, do número de pacientes-dias do BAR/BI, proveniente do censo hospitalar diário, realizado pelo Serviço de Estatística do Departamento de Informação e Documentação do IFF (FIOCRUZ, 1994b).

Foram também coletados dados de distribuição temporal de utilização dos antiinfecciosos, por evento de utilização.

\section{Análise dos dados}

Os dados brutos foram expressos em número de DDDi/100 leito-dias, calculado segundo Heckster et al., (1982):

$$
\text { DDDi } / 100 \text { leito-dias }=\frac{\text { no de DDDi x } 100}{\text { no de paciente-dias }}
$$

Por outro lado, examinou-se a diversidade dos antiinfecciosos utilizados, definindo-a como o grau de entropia da utilização e empregando os seguintes critérios: a) antiinfeccioso de uso contínuo - aqueles que foram utilizados em todos os meses do semestre;

b) antiinfecciosos de uso intermitente aqueles que não foram utilizados em pelo menos um mês do semestre;

c) evento de utilização - utilização de dado antiinfeccioso em dado mês (independente de quantidade);

d) freqüência de utilização - somatório dos eventos de utilização.

Procedimentos de análise de série temporal foram realizados em três conjuntos de dados: (1) Consumo mensal de antiinfecciosos, em DDDi/100 leito-dias (Figura 1); (2) Número de antiinfecciosos utilizados por mês, no período (Figura 2); (3) Número de antiinfecciosos de uso contínuo e uso intermitente e seus porcentuais (Tabela 2) e, como subproduto, a freqüência de utilização de antiinfecciosos de uso contínuo e de uso intermitente, por semestre (Figura 3), calculada a partir do número de eventos de utilização. 
Figura 1

Perfil e tendência de consumo (semimedianas). Berçário de Alto Risco e Berçário Intermediário,

Instituto Fernandes Figueira, janeiro de 1990 a dezembro de 1994.

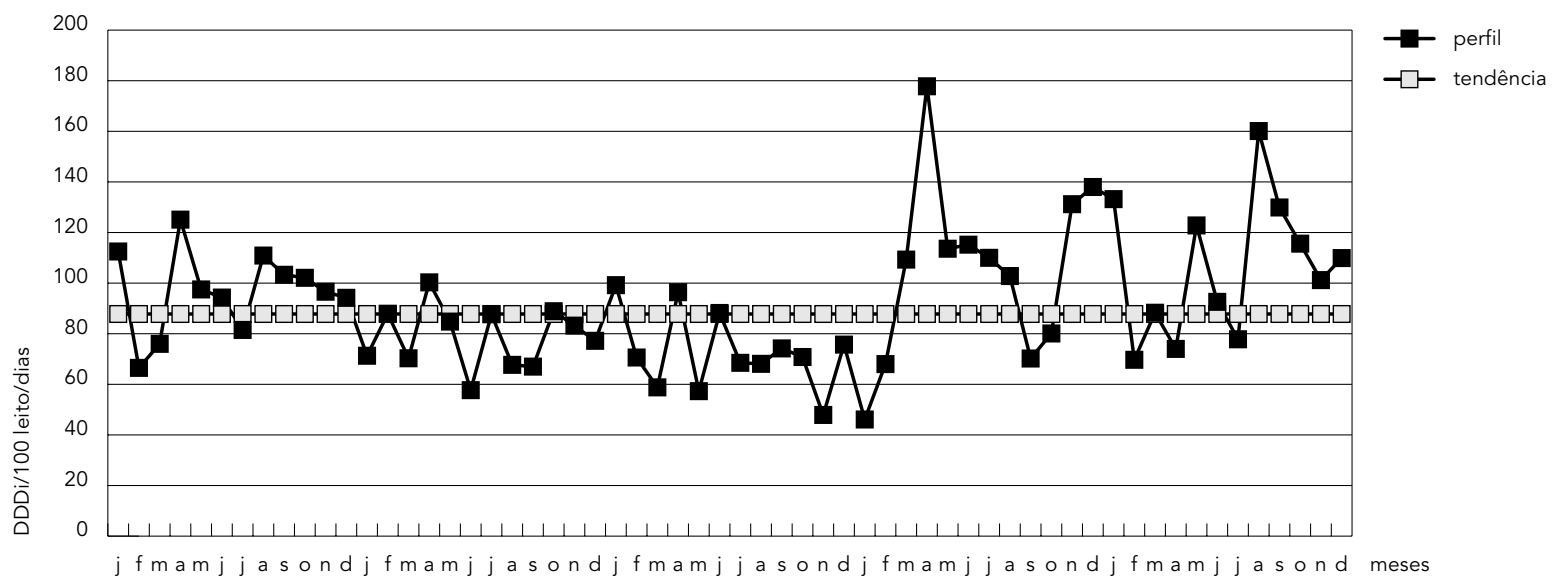

Figura 2

Perfil e tendência de diversidade (semimedianas). Berçário de Alto Risco e Berçário Intermediário, Instituto Fernandes Figueira, janeiro de 1990 a dezembro de 1994.

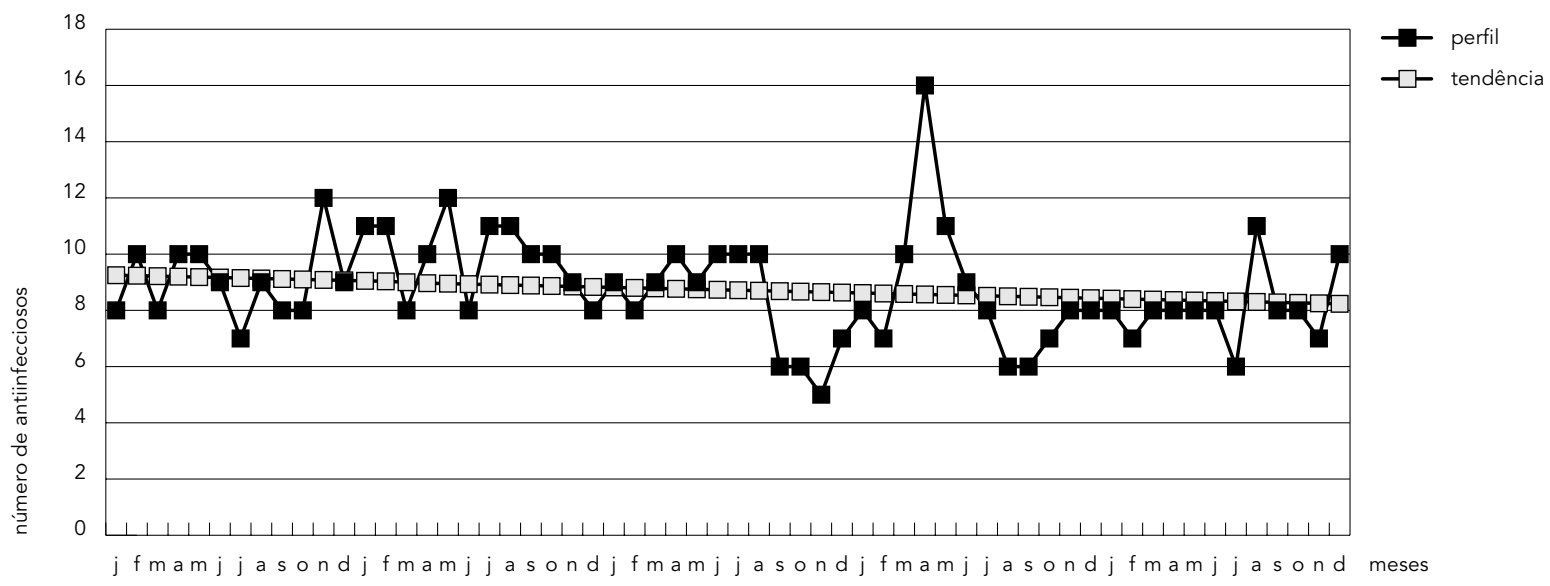


Tabela 2

\begin{tabular}{|c|c|c|c|c|c|c|}
\hline \multirow[t]{2}{*}{ Semestre } & \multicolumn{2}{|c|}{$\begin{array}{l}\text { Antiinfecciosos } \\
\text { de uso contínuo }\end{array}$} & \multicolumn{2}{|c|}{$\begin{array}{l}\text { Antiinfecciosos de } \\
\text { uso intermitente }\end{array}$} & \multirow[t]{2}{*}{ Total } & \multirow[t]{2}{*}{$\mathrm{C} \% / \mathrm{I} \%$} \\
\hline & $n$ & $\%$ & $\mathrm{n}$ & $\%$ & & \\
\hline Janeiro-junho/1990 & 4 & 30,8 & 9 & 69,2 & 13 & 0,45 \\
\hline Julho-dezembro/1990 & 4 & 26,7 & 11 & 73,3 & 15 & 0,36 \\
\hline Janeiro-junho/1991 & 5 & 26,3 & 14 & 73,7 & 19 & 0,36 \\
\hline Julho-dezembro/1991 & 5 & 35,7 & 9 & 64,3 & 14 & 0,56 \\
\hline Janeiro-junho/1992 & 5 & 35,7 & 9 & 64,3 & 14 & 0,56 \\
\hline Julho-dezembro/1992 & 4 & 28,6 & 10 & 71,4 & 14 & 0,4 \\
\hline Janeiro-junho/1993 & 5 & 26,3 & 14 & 73,7 & 19 & 0,35 \\
\hline Julho-dezembro/1993 & 5 & 41,7 & 7 & 58,3 & 12 & 0,72 \\
\hline Janeiro-junho/1994 & 5 & 38,5 & 8 & 61,5 & 13 & 0,63 \\
\hline Julho-dezembro/1994 & 5 & 38,5 & 8 & 61,5 & 13 & 0,63 \\
\hline
\end{tabular}

$\mathrm{C} \% / / \%=$ porcentagem de contínuos/porcentagem de intermitentes.

Finalmente, a diversidade e a intensidade do consumo foram examinados com base na história recente da instituição como um todo, e do BAR/BI em particular.

\section{Resultados}

\section{Observação direta das unidades}

e entrevistas - caracterizando o serviço

As equipes médica e de enfermagem desenvolviam o trabalho de forma integrada. O Corpo Clínico estava disponível 24 horas por dia, com visita diária. As reuniões da equipe de assistência eram freqüentes, o comparecimento dos residentes às sessões de supervisão, rotineiro. Nelas eram revisados os protocolos de conduta clínica da equipe e seu conhecimento acumulado. Vale frisar que a equipe médica responsável pelo BAR/BI trabalhava em conjunto desde 1989-1990 no IFF. O início do período de coleta dos dados coincide com o começo da formação da equipe.

A revisão dos prontuários acontecia em dois momentos: diariamente, pelos plantonistas que revisavam a conduta e a evolução dos pacientes, e por ocasião da alta hospitalar, pela chefia, por meio do exame da folha de resumo de alta e do restante do prontuário. Essa atividade facilitava a elaboração de estatísticas internas e aplicação de indicadores de qualidade de assistência.

A assistência prestada pelo BAR/BI sempre foi de caráter terciário, desde o início das ativi- dades, quando essas enfermarias recebiam larga parcela de pacientes externos. Em 1992, todavia, o nível de especialização dos serviços prestados sofreu incremento. O Departamento de Obstetrícia passou a direcionar a admissão na maternidade a gestantes de risco, recebendo majoritariamente pacientes oriundas do ambulatório de pré-natal do IFF. Crianças de risco, nascidas de mães de risco na maternidade do IFF, tornaram-se assim os principais pacientes do BAR e do BI.

A conduta da equipe, no que se refere a antiinfecciosos, sofreu modificações no período 1990-1994, em função de opção por fármacos de menor risco e da modificação do perfil epidemiológico do BAR/BI. Até 1990, havia predominância de hemoculturas para Klebsiella e Acinetobacter, sendo a conduta de primeira linha ampicilina e gentamicina, e de ceftriaxona e oxacilina na segunda linha. Como medida de segurança terapêutica, em 1991, a amicacina substituiu a gentamicina, promovendo uma modificação do perfil epidemiológico. Nas hemoculturas passou a predominar Staphyloccocus (Sant'anna et al., 1991). A partir daí, a segunda linha vem incluindo a vancomicina.

\section{O perfil de utilização dos antiinfecciosos de uso contínuo e intermitente no período}

A Figura 1 mostra tendência de aumento de consumo no final do período, que, embora discreta, representa mudança da ordem de 20 DDDi/100 leito-dias na unidade. O consumo apresenta flutuações, mormente a partir de 
Freqüências de utilização (percentuais) de antiinfecciosos de uso contínuo e de uso intermitente por semestre.

Berçário de Alto Risco e Berçário Intermediário, Instituto Fernandes Figueira, janeiro de 1990 a dezembro de 1994.

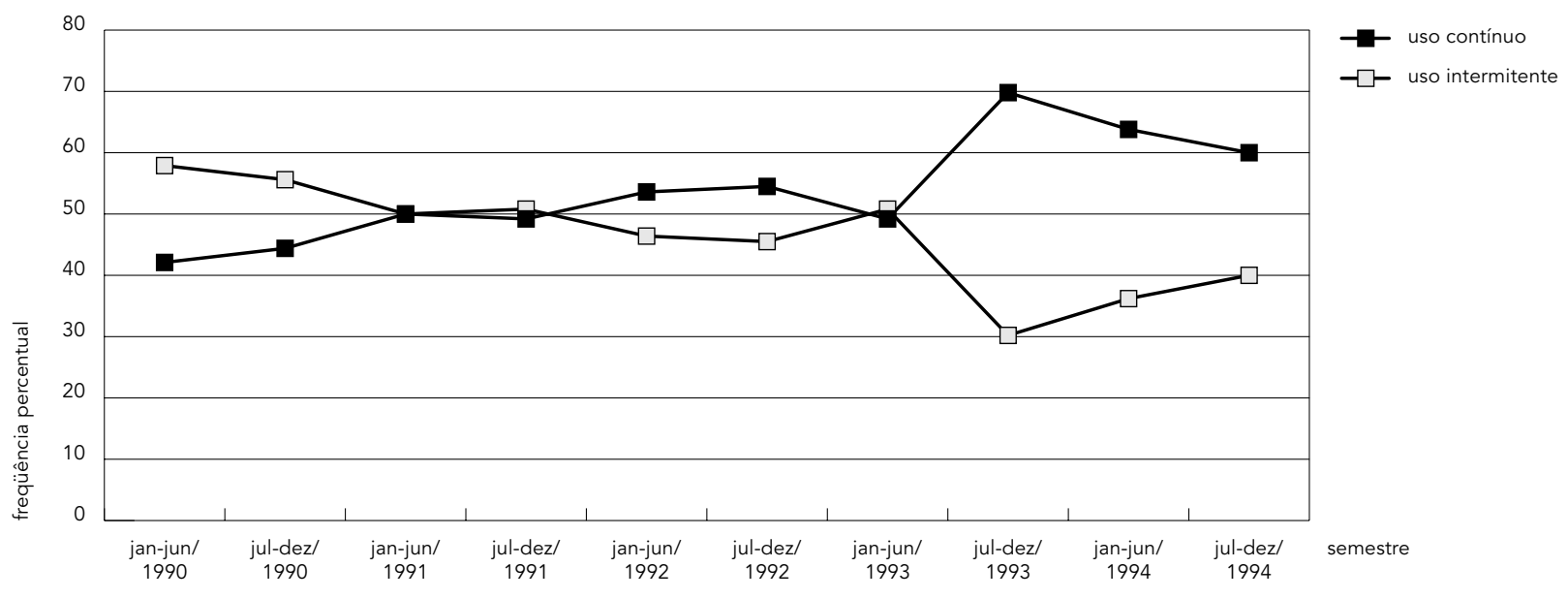

abril de 1993, sem padrão sazonal evidenciado. A pesquisa documental permitiu determinar a ocorrência de desabastecimento, por restrição orçamentária, no final de 1992. A Figura 1 evidencia o fenômeno. Os registros do BAR/BI informam ainda a presença de diversos casos de crianças em estado grave, com ventilação assistida, em dezembro de 1992 e abril e julho de 1993. Nota-se acréscimo considerável do consumo a partir do início de 1993. A Figura 2 ilustra a diminuição na diversidade de antiinfecciosos consumidos no tempo de estudo como um todo. A comparação entre as figuras 1 e 2 , permite identificar que o período que corresponde ao menor consumo é também aquele em que ocorre a menor diversidade de uso. No período que se segue, observa-se simultaneamente o aumento da diversidade e do consumo de antiinfecciosos.

A Tabela 2 mostra o porcentual de antiinfecciosos de uso intermitente e contínuo em cada semestre, durante o período. Nota-se que a razão entre porcentagens de antiinfecciosos de uso contínuo (C\%) e de uso intermitente (I\%) sofre flutuação no decorrer do período. Cresce a utilização dos de uso contínuo enquanto que diminui a utilização daqueles de uso intermitente. Essa tendência pode ser claramente observada ao examinar-se as freqüências porcentuais de antiinfecciosos de uso contínuo e intermitente por semestre; elas sofrem inversão no decorrer do período, sendo o pri- meiro semestre de 1993, bastante característico do fenômeno (Figura 3).

\section{Discussão}

\section{O fornecimento como estimativa do consumo}

A estimativa do consumo por meio de dados de fornecimento está longe do ideal, não contemplando uma série de eventos e situações plausíveis na rotina hospitalar, tais como, desperdício, administração errônea, inobservância do regime terapêutico ("non-compliance") e mudanças terapêuticas tempestivas. Erros próprios do sistema de distribuição, como falhas no registro, na entrega e falha ou ausência de auditoria nos estoques das enfermarias também podem subtrair dados importantes.

A favor desse tipo de coleta, estão a rapidez e a facilidade com que ela pode ser realizada, além da possibilidade da elaboração de estudos comparativos entre serviços diferentes de uma mesma unidade de saúde (Ruiz, 1993).

Os registros de fornecimento, ao contrário dos prontuários dos pacientes, são produzidos como instrumento administrativo pela farmácia hospitalar e sofrem controle de qualidade prático: são os totais de fornecimento que corroboram as compras de medicamentos, feitas periodicamente. Erros grosseiros, como tam- 
bém os de menor monta, merecerão a atenção dos responsáveis, e sua correção, ainda que num momento posterior. É importante compreender que os dados de fornecimento não pretendem e nem podem apresentar mais do que uma visão geral da situação do consumo, deixando claro, no entanto, sua importância como ferramenta no estabelecimento do perfil de consumo dos fármacos e na análise da situação de utilização dessas tecnologias. O fornecimento atua como elemento chave para levantar hipóteses sobre o uso racional.

\section{O indicador empregado}

A DDD é uma metodologia que permite a visualização dos padrões de consumo de medicamentos no decorrer do tempo, principalmente quando se deseja, segundo Hekster et al. (1982), uma "estimativa rápida". Utilizada na obtenção de uma taxa, como DDD/100 leitodias, torna possível a verificação de oscilações reais de consumo, independentes do crescimento da população ou do número de pacientes. Não dá conta da utilização individual de dado fármaco, mas pode informar sobre a probabilidade desse fármaco vir a ser utilizado, tendo portanto, certo valor preditivo.

O uso da DDDi tem como função a adaptação dos dados, de forma a refletir um consumo compatível com aquele de uma unidade pediátrica; um consumo de 3DDDi/100 leito-dias poderá ser melhor compreendido do que um de 0,3DDD/100. A DDDi permite multiplicar por dez o consumo anteriormente expresso em DDDs, sem prejudicar a lógica da unidade de medida ou modificar o perfil de consumo.

\section{O consumo total e a diversidade dos} antiinfecciosos utilizados - levantando hipóteses sobre fatores relacionados

Os resultados deste estudo, indicam que no período considerado, o consumo absoluto de antiinfecciosos nas unidades BAR e BI aumentou, enquanto que sua diversidade diminuiu. Também é possível perceber uma mudança no tipo dos antiinfecciosos utilizados: no início do período estudado, predominavam os de uso intermitente, observando-se o oposto ao final. É razoável portanto, afirmar que os antiinfecciosos de uso contínuo foram os maiores responsáveis pelo incremento do consumo.

Fausto et al. (1995) relatam que em 1992, o IFF passou por câmbio na assistência perinatal. Em função da grave crise que se abalava sobre o setor público de saúde no Estado do Rio de Janeiro, e por apresentar condições assis- tenciais próprias de hospital terciário, ao IFF passaram a acorrer cada vez mais pacientes graves que permaneciam por maiores períodos em setores especializados.

Esses dados foram discutidos com representantes da equipe do BAR/BI, considerando a percepção e a prática dos profissionais diretamente envolvidos no cuidado dos pacientes.

O primeiro fato mencionado pela equipe foi a integração entre os Departamentos de Obstetrícia (Maternidade) e o de Neonatologia, com a restrição de admissão na maternidade a gestantes de risco, cujo pré-natal tenha sido conduzido na instituição em 1992. Os profissionais entrevistados consideraram esse fato como gerador de incremento no nível de especialização dos serviços prestados pelo BAR/BI. $\mathrm{O}$ argumento é de que a maior quantidade de mães de risco tenha-se refletido sobre o número de neonatos de risco nascidos na instituição. $\mathrm{O}$ aumento real do consumo de antiinfecciosos estaria, portanto, estreitamente ligado às complicações por que passam muitos desses bebês no período perinatal.

Por outro lado, a equipe compreende que a diminuição da diversidade dos antiinfecciosos utilizados é reflexo de uma série de fatores. O aumento do número de bebês de risco, filhos daquelas gestantes já pacientes do IFF, diminuiu a oferta de vagas no BAR/BI para neonatos provenientes de outras instituições. Muitos desses bebês eram internados com terapêutica medicamentosa em curso, e os antiinfecciosos tinham seu uso continuado usualmente até fim do tratamento, de modo a contornar possíveis riscos associados à sua suspensão. Quando o ingresso ficou mais restrito para pacientes nascidos fora do IFF, verificou-se uma diminuição da diversidade dos antiinfecciosos já que não mais se fazia necessário dar continuidade a tratamento iniciados em outras unidades, os quais diferiam, muitas vezes, dos protocolos internos.

Considera-se como fator importante, o tempo de integração da equipe do BAR/BI, trabalhando em conjunto desde 1989-1990. Esse fator é apontado por seus representantes como um forte componente da homogeneidade das ações e procedimento firme da conduta clínica dos residentes da unidade. A supervisão constante e a coordenação de esforços, teria levado a equipe a aderir de forma crescente aos protocolos terapêuticos considerados como mais eficientes e seguros, desestimulando mudanças ou inovações percebidas como clinicamente desnecessárias ou de eficácia duvidosa.

O Departamento de Neonatologia mantinha ainda registros próprios das infecções ocorridas por tipo, ajudando a nortear a conduta 
clínica da equipe. Tal procedimento encontrase em acordo com o apontado por autores como Leibovitz et al. (1997), Natsch et al. (2000) e Gindre et al. (2000), que afirmam que a vigilância epidemiológica dentro das unidades de terapia intensiva neonatal deva fornecer subsídios para mudanças de conduta quanto ao uso de antiinfecciosos.

A conduta de primeira linha até 1991, previa o uso de ampicilina e gentamicina para fazer frente a um perfil de hemoculturas dominado por Acinetobacter e Klebsiella. A mudança de gentamicina por amicacina, obedecendo a critérios de segurança terapêutica, promoveu reflexamente a modificação de perfil, passando a pontificar culturas positivas para Staphyloccocus. A vancomicina, que até então era utilizada pontualmente, passou a ser incorporada como integrante da terapêutica de segunda linha, juntamente com a oxacilina e a ceftriaxona. Vê-se por isso, um incremento real no consumo desses antiinfecciosos durante o período.

\section{O perfil de utilização e racionalidade terapêutica}

O perfil de utilização de antiinfecciosos no BAR/BI pelo período estudado, compreendeu tanto o consumo de todos e de cada um dos fármacos empregados, quanto o grau de diversidade dessa utilização.

Dados de incidência de infecção hospitalar não estiveram disponíveis para consulta e comparação. Não obstante, as oscilações apresentadas nas figuras 1 e 2 , sustentam ainda possibilidade de explicação nessa linha. A falta de material médico e a restrição do quantitativo de medicamentos, pode ter suscitado a alteração do perfil das infecções hospitalares nas unidades, constituindo possibilidade de explicação para a necessidade de uso mais intenso e diversificado de antiinfecciosos em período subseqüente ao das faltas.

A discussão da racionalidade é complexa e engloba fatores não examinados no âmbito deste trabalho, como disponibilidade e acesso, garantia de qualidade do medicamento em todas as etapas do abastecimento, dispensação adequada e cumprimento do regime terapêutico, dose e duração de tratamento. O que se pode argumentar, contudo, é que um cenário com menor número de fármacos facilita o trabalho na direção do uso racional, seja pela simplicidade de suprimento e de controle da qualidade do medicamento, seja pela segurança no emprego de metodologias adequadas para controle de qualidade da prescrição, da distribuição e do próprio uso.
Ainda, os resultados do estudo, se examinados separadamente, não permitem considerações mais elaboradas sobre a questão do uso racional. Aumento real de consumo significa que o contingente de pacientes internados usou crescente quantidade de medicamento no decorrer do período. Isolado do contexto da unidade hospitalar, esse fato poderia depor contra uma possível tendência racionalizadora. Por outro lado, a redução da diversidade dos antiinfecciosos utilizados, per se, também não implicaria necessariamente em uso mais racional. As necessidades individuais dos pacientes em muitas situações ditam utilização diversificada, e nem por isso injustificada.

Uma vez que, a racionalidade se traduz na avaliação de disponibilidades e oportunidades, riscos e benefícios, devemos analisar os dados numéricos à luz do contexto institucional. Observa-se o ingresso crescente de pacientes com quadros de maior risco, justapondo-se a aumento do consumo de antiinfecciosos. Em contrapartida, a crescente integração da equipe traz refinamento e ajuste de condutas terapêuticas, e adesão consensual a protocolos de tratamento. Desse modo, o aumento real no consumo de antiinfecciosos, poderia constituir reflexo da crescente gravidade dos casos atendidos, enquanto que a restrição de sua variedade poderia ser conseqüência de conduta clínica mais homogênea da equipe.

\section{Considerações finais}

A resposta da unidade estudada à demanda externa de atendimento terciário, foi indiretamente a "construção" de novo perfil da assistência perinatal, completando-se pela constância de composição e integração da equipe de profissionais de saúde, dentro da UTI neonatal e com a Obstetrícia, e pela adoção crítica de procedimentos terapêuticos. Ao contextualizar os eventos e relacionar dados quantitativos com os qualitativos, foi possível apontar que as mudanças institucionais foram fatores importantes na modificação do perfil de utilização de antiinfecciosos.

É influente a visão de que no emprego mais judicioso e coerente de menor número de fármacos, encontramos o caminho da racionalidade terapêutica. Em 1982, Hekster e colaboradores, realizando um estudo ao longo de cinco anos, mostraram mudanças no perfil de utilização de antiinfecciosos, motivadas por intervenção nas condutas de prescrição, com incremento do uso racional. Ao longo dos últimos vinte anos, esse princípio, primeiramente pro- 
posto pela OMS, tem-se difundido e consolidado (Osorio-de-Castro et al., 2000).

A utilização de medicamentos no decorrer do tempo em uma unidade de saúde, região ou país é um processo dinâmico e lábil. Por meio dos EUM pode-se examinar tanto a história da utilização e dos fatores que sobre ela influem, quanto seu perfil. Esse, por sua vez, indica tendências e possui certo valor de predição. A aná-

\section{Referências}

ALBRANT, D. H., 1999. AphA drug treatment protocols: Management of pediatric convulsive status epilepticus. Journal of the American Pharmaceutical Association, 39:469-676.

ANAND, K. J. S.; ABU-SAAD, H. H.; AYNSLEY-GREEN, A.; BANCALARI, E.; BENINI, F.; CHAMPION, G. D.; CRAIG, K. D.; DANGEL, T. S.; FOURNIERCHARIÈRE, E.; FRANCK, L. S.; GRUNAU, R. E.; HERTEL, S. A.; JACQZ-AIGRAIN, E.; JORCH, G.; KOPELMAN, B. I.; KOREN, G.; LARRSON, B.; MARLOW, N.; MCINTOSH, N.; OHLSSON, A.; OLSSON, G.; PORTER, F.; RICHTER, R.; STEVENS, B. \& TADDIO, A., 2001. Consensus statement for the prevention and management of pain in the newborn. Archives of Pediatrics and Adolescent Medicine, 155:173-180.

ANDERSEN, C. T. \& MEBERG, A., 1999. Drug use in a neonatal unit. Tidsskr Nor Saegeforen, 119:197200.

CAPELLÀ, D., 1993. Descriptive tools and analysis. In: Drug Utilization Studies. Methods and Uses (M. N. G. Dukes, ed.), pp. 55-78, Copenhagen: World Health Organization Regional Office for Europe.

FAUSTO, M. C. R.; PINTO, R. F. \& MAGNO, A., 1995. Gestação de Risco: Construindo o Perfil das $\mathrm{Mu}$ lheres que Procuram o Pré-natal do Instituto Fernandes Figueira. Monografia de Especialização, Rio de Janeiro: Escola Nacional de Saúde Pública, Fundação Oswaldo Cruz.

FIJN, R.; ENGELS, S. A. G.; BROUWERS, J. R. B. J.; KNAAP, R. J. \& DE JONG-VAN den BERG, L. T. W., 2000. Dutch hospital drug formularies: Pharmacotherapeutic variation and conservatism, but concurrence with national pharmacotherapeutic guidelines. British Journal of Clinical Pharmacology, 49:254-263.

FISCHER, J. E.; RAMSER, M. \& FANCONI, S., 2000. Use of antibiotics in pediatric intensive care and potential savings. Intensive Care Medicine, 26: 959-966.

FUCHS, F. D.; KLAG, M. J. \& WHELTON, P. K., 2000. The classics: A tribute to the fiftieth anniversary of the randomized clinical trial. Journal of Clinical Epidemiology, 53:335-342. lise conjunta desses componentes, mediante confronto com a melhor evidência disponível, pode levar a reflexões e considerações quanto à racionalidade dessa mesma utilização.

No presente trabalho foi examinado o perfil de utilização de medicamentos em unidade neonatal, face a seus determinantes e à luz do paradigma do uso racional de medicamentos.

FIOCRUZ (Fundação Oswaldo Cruz), 1994a. Relação de Medicamentos Totalizados por Área. Rio de Janeiro: Serviço de Farmácia, Departamento de Assistência, Instituto Fernandes Figueira, FIOCRUZ.

FIOCRUZ (Fundação Oswaldo Cruz), 1994b. Movimentação Hospitalar. Rio de Janeiro: Serviço de Estatística, Departamento de Informação e Documentação, Instituto Fernandes Figueira, FIOCRUZ.

GINDRE, I.; MAISONNEUVE, H.; RICHE, B. \& DUROCHER, A., 2000. Guidelines for good clinical practice: Use of antibiotics in 207 public and private French hospitals in 1999. Presse Medicale, 29:1807-1812.

HEKSTER, Y. A.; VREE, T. B.; GORIS, R. J. A. \& BOEREMA, J. B. J., 1982. The defined daily dose per 100 bed-days as a unit of comparison and a parameter for studying antimicrobial drug use in a university hospital. Journal of Clinical and Hospital Pharmacy, 7:251-260.

JACOBS, R. F., 2000. Judicious use of antibiotics for common pediatric respiratory infections. Pediatric Infectious Diseases Journal, 19:938-943.

KOHN, L. T., 2001. The Institute of Medicine report on medical error. Overview and implications for pharmacy. American Journal of Health-Systems Pharmacy, 58:63-66.

LAPORTE, J.-R.; BAKSAAS, I. \& LUNDE, P. K. M., 1993. General background. In: Drug Utilization Studies. Methods and Uses (M. N. G. Dukes, ed.), pp. 5-22, Copenhagen: World Health Organization Regional Office for Europe.

LEIBOVITZ, E.; FLIDEL-RIMON, O.; JUSTER-REICHER, A.; AMITAY, M.; MISKIN, A.; BARAK, Y. \& MOGILNER, B., 1997. Sepsis at a neonatal intensive care unit: A four-year retrospective study (19891992). Israeli Journal of Medical Science, 33:734-738.

MATHESON, I., 1991. Epidemiological and Pharmacokinetic Studies on Drugs and Breast-Feeding. Oslo: University of Oslo.

MOSEGUI, G. B. G., 1999. Avaliação da qualidade do uso de medicamentos em idosos. Revista de Saúde Pública, 33:444-457.

NATSCH, S.; KULLBERG, B. J.; MEIS, J. F. G. M. \& VAN DER MEER, J. W. M., 2000. Earlier initiation of an- 
tibiotic treatment for severe infections after interventions to improve the organization and specific guidelines in the emergency department. Archives of Internal Medicine, 160:1317-1320.

OSORIO-DE-CASTRO, C. G. S.; MOSEGUI, G. B. G.; PEIXOTO, M. A. P.; CASTILHO, S. R. \& LUIZA, V. L., 2000. Estudos de Utilização de Medicamentos. Rio de Janeiro: Editora Fiocruz.

POLLACK, L. D.; RATNER, I. M. \& LUND, G. C., 1998. United States Neonatology Practice Survey: Personnel, practice, hospital, and neonatal intensive care unit characteristics. Pediatrics, 101:398-405.

PONS, G.; LASSALE, C. \& ESCHWEGE, E., 1999. How to improve drug development and utilization in pediatrics. Therapie, 54:423-432.

RUIZ, I., 1993. Estudios de utilización de medicamentos. In: Fundamentos de Farmacia Clínica (A. Arancíbia, E. Cid, C. Domecq, C. Paulos, M. Pezzani, E. Pinilla, M. Rojas, I. Ruiz \& M. Vargas, org.), pp. 299-308, Santiago de Chile: PIADE/Universidad de Chile.

SACKETT, D. L.; STRAUS, S. E.; RICHARDSON, W. S.; ROSENBERG, W. \& HAYNES, R. B., 2000. EvidenceBased Medicine. How to Practice and Teach EBM. Toronto: Churchill Livingstone.

SANT'ANNA, G. M.; MARTINS, F. F.; ANDRADELOPES, J. M. \& CARVALHO, M., 1991. Impacto da introdução de uma nova rotina de antibioticoterapia na prevalência de microorganismos de uma UTI neonatal. In: Congresso Brasileiro de Pediatria, Anais, p. 155. Porto Alegre: Sociedade Brasileira de Pediatria.
SCHNEIDER, P. J., 1999. Creating an environment for improving the medication-use process. American Journal of Health-Systems Pharmacy, 56:17691772.

SCHNEIDER, P. J., 2001. Pharmacists building a safer health system. American Journal of Health-Systems Pharmacy, 58:66-68.

TOGNONI, G. \& LUNDE, P. K. M., 1993. Therapeutic formularies, essential drugs, drug utilization studies. In: Drug Utilization Studies. Methods and Uses (M. N. G. Dukes, ed.), pp. 43-53, Copenhagen: World Health Organization Regional Office for Europe.

WHO (World Health Organization), 1987. The Rational Use of Drugs. Report of the Conference of Experts. Geneva: WHO.

WHO (World Health Organization), 1993. Anatomical Therapeutic Chemical (ATC) Classification Index - Including Defined Daily Doses (DDDs) for Plain Substances. Oslo: WHO.

WHYTE, S.; BIRRELL, G. \& WYLLIE, J., 2000. Premedication before intubation in UK neonatal units. Archives of Diseases in Children Fetal and Neonatal Edition, 82:F38-F41.

Recebido em 23 de abril de 2001

Versão final reapresentada em 16 de julho de 2001

Aprovado em 1 de agosto de 2001 